\title{
Poor description of non-pharmacological interventions: analysis of consecutive sample of randomised trials
}

\author{
(9) $\Theta$ OPEN ACCESS
}

\section{Tammy C Hoffmann associate professor of clinical epidemiology, Chrissy Erueti assistant professor, Paul P Glasziou professor of evidence-based medicine}

Centre for Research in Evidence-Based Practice, Faculty of Health Sciences and Medicine, Bond University, Qld, Australia, 4229

\begin{abstract}
Objectives To evaluate the completeness of descriptions of non-pharmacological interventions in randomised trials, identify which elements are most frequently missing, and assess whether authors can provide missing details.

Design Analysis of consecutive sample of randomised trials of non-pharmacological interventions.

Data sources and study selection All reports of randomised trials of non-pharmacological interventions published in 2009 in six leading general medical journals; 133 trial reports, with 137 interventions, met the inclusion criteria.

Data collection Using an eight item checklist, two raters assessed the primary full trial report, plus any reference materials, appendices, or websites. Questions about missing details were emailed to corresponding authors, and relevant items were then reassessed.

Results Of 137 interventions, only 53 (39\%) were adequately described; this was increased to 81 (59\%) by using 63 responses from 88 contacted authors. The most frequently missing item was the "intervention materials" (47\% complete), but it also improved the most after author response (92\% complete). Whereas some authors (27/70) provided materials or
\end{abstract}

further information, other authors (21/70) could not; their reasons included copyright or intellectual property concerns, not having the materials or intervention details, or being unaware of their importance. Although 46 $(34 \%)$ trial interventions had further information or materials readily available on a website, many were not mentioned in the report, were not freely accessible, or the URL was no longer functioning.

Conclusions Missing essential information about interventions is a frequent, yet remediable, contributor to the worldwide waste in research funding. If trial reports do not have a sufficient description of interventions, other researchers cannot build on the findings, and clinicians and patients cannot reliably implement useful interventions. Improvement will require action by funders, researchers, and publishers, aided by long term repositories of materials linked to publications.

\section{Introduction}

Secret remedies—branded drugs whose ingredients were kept secret-were once common, until successful campaigns in the United States and United Kingdom in the early 20th century required labels to include all ingredients. ${ }^{1}$ This policy allowed independent evaluation of treatments and provided clinicians

Correspondence to: T Hoffmann thoffmann@bond.edu.au

Extra material supplied by the author (see http://www.bmj.com/content/347/bmj.f3755?tab=related\#webextra) Supplementary figure

Video on bmj.com (see also http://bmj.com/video)

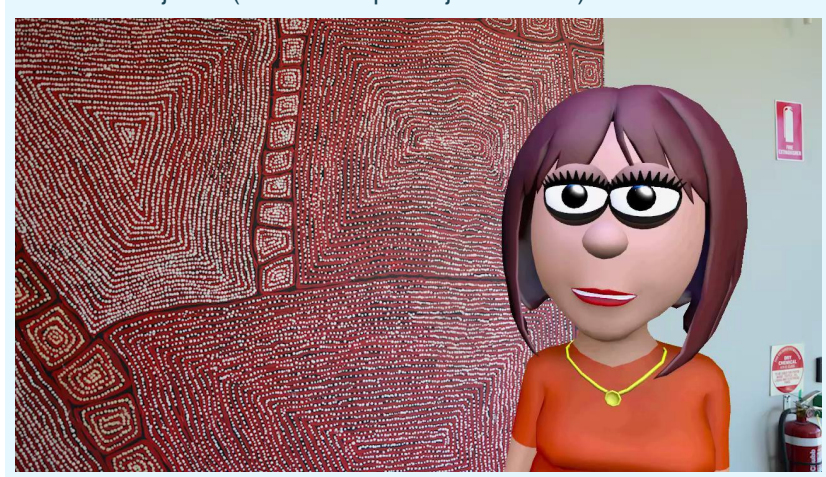


and consumers with the means to understand what they were considering using.

However, a treatment consists of more than the list of ingredients: the dose, frequency, monitoring, titration, mode of delivery, and duration of use can all influence efficacy and safety. Such details are often not well described in trials. For example, a recent analysis found that only $11 \%$ of 262 trials of cancer chemotherapy provided complete details of the trial treatments. ${ }^{2}$ The most common missing elements were dose adjustment and pre-medications, but $16 \%$ omitted even the route of administration of the drug. The completeness of descriptions of treatments may be worse for non-pharmacological interventions: one analysis found that $67 \%$ of drug treatment descriptions were adequate compared with only $29 \%$ of non-drug treatments. However, this analysis also included systematic reviews, which were less informative on average than were reports of individual trials. ${ }^{3}$

Unlike the bogus "secret remedies" of the 19th century, the current incomplete descriptions of interventions are likely to arise from poor communication and lack of awareness of the matter among authors and lack of attention by reviewers and editors. However, the consequences are similar: other researchers cannot replicate and build on research findings, and clinicians and patients cannot reliably adopt interventions shown to be useful.

To better understand what elements are most frequently missing from descriptions, and whether this is remediable, we aimed to assess the completeness of descriptions of non-pharmacological interventions in reports of trials published in major general medical journals, using a checklist to evaluate the completeness, and to assess whether missing details can be obtained by contacting authors of trial reports.

\section{Methods}

\section{Search strategy and selection of reports of trials}

We included all reports of randomised controlled trials of non-pharmacological interventions published in 2009 in one of the six leading general medical journals (based on ISI Web of Knowledge impact factor for 2010): New England Journal of Medicine, JAMA, Lancet, Annals of Internal Medicine, PLOS Medicine, and $B M J$. We defined non-pharmacological interventions as interventions including surgery, technical procedures, devices, rehabilitation, psychotherapy, behavioural interventions, and complementary and alternative medicine. ${ }^{4}$

We excluded reports if they evaluated a pharmacological intervention, a screening programme, or a diagnostic technique (for example, endoscopy) or if they reported secondary analyses, such as an economic analysis of a non-pharmacological intervention trial for which the main results had been published elsewhere or before 2009. Reports were eligible if a non-pharmacological intervention had been compared with a pharmacological intervention or when a complex intervention included a pharmacological component if the major focus was a non-pharmacological component. When reports contained evaluations of more than one non-pharmacological intervention (such as by using three groups or a factorial design), we rated the descriptions of each intervention separately.

An experienced medical librarian searched PubMed in April 2011, using the restrictions of year (2009), publication type ("randomized controlled trial"), and journal title (the six chosen journals). Two authors (TH and PG) screened the 358 titles and abstracts retrieved, identified reports that might meet the inclusion criteria, and retrieved the complete and unabridged reports $(n=138)$. Disagreements about unclear eligibility were resolved by discussion.

\section{Rating of intervention descriptions}

Before rating the reports in our study sample, we rated a sample of reports that met our eligibility criteria but had been published in 2008, discussed responses, and continued rating reports until high agreement was reached. Two authors (TH and PG) independently used a checklist to rate the description of the interventions in each eligible report. The checklist is based on the work of Davidson and colleagues and the CONSORT extension statement for non-pharmacological interventions and has been used, in a slightly modified form, in a previous study. ${ }^{4-6}$ The checklist contains eight items (table $1 \Downarrow$ ). Seven of the items are rated as "Yes" (indicating that the element of the intervention had been clearly described) or "No" (not reported or not clearly described). Each item in the checklist had an explanatory statement that guided the raters in the scope and interpretation of the item. For "No" responses, we recorded detailed comments about what was missing. The eighth item was an overall question: "Is the description of the intervention complete?" Reports could score a "Yes" on this item only if all other items had been rated as "Yes." We also routinely and systematically assessed whether the intervention details might have been described further in other sources (such as websites, online appendices, or reference materials). If so, we used these additional sources when completing the checklist items. Disagreements in ratings were resolved by discussion, with reference to the explanatory statement for the relevant item/s, between the two raters.

For all reports with any checklist items rated as having an incomplete description, the raters generated a list of questions about element/s of the intervention that were missing or unclear. We emailed these questions to the corresponding author. If no response was received within four weeks, we sent up to two reminder emails at four weekly intervals. When responses were received from authors, the raters collaboratively re-rated the relevant items as either "Yes-clear after author reply" or "No-not clear after author reply."

\section{Data analysis}

We entered initial and follow-up ratings of reports and questions emailed to authors into a customised database. We used Excel to analyse data descriptively.

\section{Results}

Of the 138 reports for which we were able to obtain full texts, we excluded five (three economic evaluations and two long term follow-up results) and four reports contained evaluations of two non-pharmacological interventions (see supplementary figure). Our final sample thus contained published reports of 137 interventions from 133 trials. Table $2 \Downarrow$ shows the journals in which the reports had been published and the categories of intervention that had been evaluated. ${ }^{7}$

\section{Completeness of intervention descriptions}

Figure $1 \Downarrow$ shows, for each of the checklist items, the percentage of interventions that were clearly described in primary reports and after reply from authors of trial. Overall, 53 (39\%) of the interventions were adequately described in primary reports, and this increased to $81(59 \%)$ after contact with authors. The checklist item about intervention materials scored most poorly 
in primary reports (complete in 64 (47\%) of interventions) but was also the item that showed the most improvement (complete in $126(92 \%))$ after reply from trial authors.

We sent questions to authors of reports about 88 of the interventions (from 84 trials), with a mean of 2.7 (SD 1.5, range 1-6) questions per author. We received no response from 25 authors after two reminders. On the basis of the responses from the 63 authors who replied, the overall rating changed from "No" to "Yes" for 29 of the interventions and remained as "No-description not complete" for 34 interventions (for example, requested materials were not sent or the response did not clearly answer the question). Table $3 \Downarrow$ provides verbatim examples of incomplete reporting of descriptions of interventions from the primary reports.

\section{Process of obtaining complete description of intervention-an example}

Figure $2 \Downarrow$ illustrates the key steps in the study methods and the process of obtaining a complete description of an intervention for one of the included reports. After the initial rating of this report, ${ }^{8}$ four items - setting, provider, procedure, and materials-were assessed as incompletely described, so the overall rating item ("Is the overall description complete?") was "No." We emailed the corresponding author with five questions (shown in fig $2 \Downarrow$ ). On the basis of the responses, each of these items and the overall rating were changed to "Yes." By contacting the author, we also learnt that even though the trial used a DVD that was not available, an iPhone app (in Dutch only) containing the exercises and exercise schedule was now available, as was a YouTube clip of the exercises. However, neither of these resources was mentioned in the 2009 report.

\section{Materials used in interventions}

Most of the questions that were sent to authors were about materials used in the intervention. We emailed 70 authors with at least one question about materials and received responses from 27 authors that enabled the rating of the materials item to be changed to "Yes." Typically this was because authors sent copies of the materials used (for example, written materials used with study participants, such as patient education materials or staff training materials) or provided information about how to access intervention materials or further details (such as a website or journal article that was not referenced in the primary report). Of the other 21 authors who responded, the rating for the materials item, and the overall rating, remained as "No." The box shows typical reasons for this.

\section{Further intervention information contained on websites}

For one third $(\mathrm{n}=46)$ of the interventions, a relevant website existed that contained further information about the intervention or at least some of the materials used in the intervention. Only $35(76 \%)$ of these websites were mentioned in the report and, of these, just over half $(19 ; 54 \%)$ were freely accessible. Many websites were behind a paywall, and for others the website address provided in the report no longer existed. Several interventions had relevant websites, but we learnt of these only by contacting the author or through internet search engines. Figure $3 \Downarrow$ summarises these websites according to how we found out about the website's existence (for example, mentioned in the original report, email from author), whether any website links provided worked, and whether the site content was accessible free of charge.

\section{Discussion}

More than half (61\%) of the interventions assessed in this study were not described in sufficient detail in the published primary report to enable replication of the intervention in practice. This problem is partly remediable: a third of the incompletely described interventions could be completed by contacting study authors for further information. Obtaining this additional information took some effort: compilation of omissions, up to three emails, and subsequent piecing together of information from disparate sources. Clinicians wishing to use an intervention in practice are unlikely to invest this amount of time in obtaining the necessary details and materials. Even for trials in which the intervention was not effective, complete descriptions of interventions are important for other groups of research users. For example, this includes other researchers (who may wish to build on the trial or modify the intervention in some way) and systematic reviewers (who need details of intervention to assist with assessing and understanding heterogeneity).

Most interventions included some materials, such as written materials for educating patients or materials for training staff, without which the interventions cannot be used in practice. Despite their importance, just over half (53\%) of the reports neither sufficiently described these materials nor gave details about how to obtain copies. Consequently, questions about materials were our most common question to authors. Comments from some authors suggested a lack of awareness of the importance of making intervention materials available. Other authors were reluctant to make materials available publicly (often owing to real or perceived concerns about copyright or intellectual property). Sometimes, corresponding authors no longer had copies of the materials or were uncertain about intervention details.

Several previous studies have documented deficiencies in the descriptions of interventions: an analysis of patient education interventions found that only $17 \%$ were replicable, with information about the content of sessions most commonly missing ${ }^{9}$; none of 11 trials of music therapy provided all necessary details $\mathrm{s}^{10}$; and an analysis of 158 surgical studies found that only $41 \%$ provided some details of the surgical procedure. ${ }^{11}$ However, only one previous study seems to have included writing to authors to obtain additional information; a checklist was not used, and only a small sample of non-pharmacological interventions was included. ${ }^{3}$

\section{Recommendations}

The omission of essential information about interventions is a substantial, yet remediable, contributor to the enormous worldwide waste in research funding that occurs because research is unpublished or unusable. ${ }^{12}$ Reducing this waste will require action by funders, researchers, and publishers at multiple stages, including pre-submission, editorial review, and publication. However, missing details in the reports of intervention studies is part of a wider problem of non-replicable interventions in trials and systematic reviews. This has been the topic of a consensus meeting, ${ }^{13}$ which made several recommendations. Two of these are relevant to the problems identified in this research.

A key recommendation was that: "The reporting standards for interventions in trials (CONSORT, etc) and systematic reviews (PRISMA) should be improved and standardised (specific checklists)." The CONSORT extension statement for the reporting of randomised trials of non-pharmacologic treatment contains four intervention related items: precise details of the intervention, description of the intervention components (and 


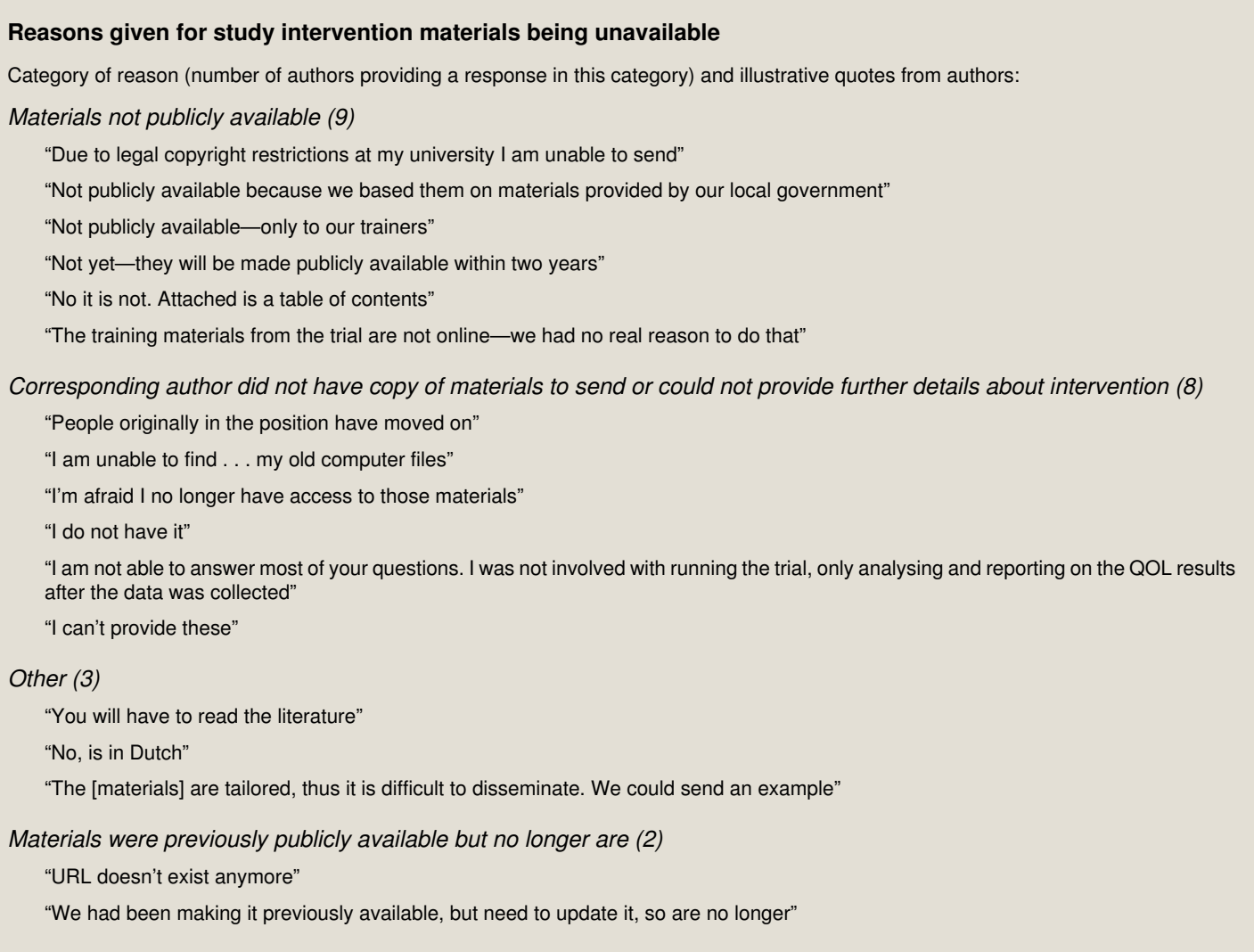

where applicable, description of tailoring procedures), details of how interventions were standardised, and details of how adherence to the protocol by care providers was assessed. ${ }^{4}$ However, this CONSORT extension does not mention intervention materials, which we found to be the most poorly reported, and remediable, element. The mere existence of an appropriately detailed checklist is unlikely to be sufficient to improve quality of reporting. Few journals request the use of extension statements, and, even when endorsed by journals, subsequent adherence to them by published reports does not necessarily occur. ${ }^{14}$ Journal editors and reviewers have a responsibility to be aware of the importance of complete reporting of non-pharmacological interventions and to implement policies and processes to ensure that this occurs.

A second recommendation was that: "A stable 'intervention bank' should be established (e.g. videos, manuals, and fidelity tools linked to trial registration number) to overcome the problem of word restrictions in journals, etc." Although hosting intervention materials on websites may seem to be a logical way of making them available, problems such as maintaining operational sites were already apparent in this sample of quite recent reports. One striking finding was that a third of the trials had a website with additional intervention information/materials, but a quarter of these were not mentioned and many were not freely accessible or the URL no longer worked. This suggests that responsibility for maintaining websites that contain intervention details should not lie solely with journals, authors, or their institutions, but instead with organisations that have greater stability and longevity. Similar to drug treatments in pharmacopoeias, an equivalent compilation for non-drug interventions is needed. ${ }^{1516}$

\section{Strengths and limitations of study}

The strengths of this study include the wide range of non-pharmacological interventions, duplicate rating, and the verification of missing details and materials with authors.
However, $28 \%$ of authors did not respond, limiting the completeness of this verification. A further limitation is that we selected reports only from leading general medical journals, and findings may not be generalisable. If the quality of reporting of interventions is similar to the quality of reporting of methodological features in randomised trials, which is better in journals with a higher impact factor, ${ }^{17}$ then our study may have underestimated the size of the problem. Our study may have also found an even larger problem of poor reporting if we had assessed whether the reports had evaluated or described the fidelity of the intervention, which has been identified as an additional element that should be included in descriptions of interventions. $^{5}$

\section{Further work needed}

Further work is needed in several areas. Firstly, we need to better understand why authors do not provide more complete descriptions of interventions. Secondly, we need to develop the guidelines and tools to assist authors and editors in providing complete descriptions of interventions. Several of the extensions to the CONSORT statement have been motivated by a need to obtain better details of interventions, but these differing extensions need harmonisation to clarify what is generic and what is specific to different types of interventions. As many trials now publish a protocol, providing additional intervention detail in the published protocol may be one way of overcoming word limit restrictions in the trial's primary paper. The recently published reporting guideline for trial protocols, the SPIRIT 2013 Statement, ${ }^{18}$ contains four items (11a-d) about the reporting of the intervention. Further work is needed to ensure that the primary paper makes explicit mention of all related documents (such as protocols, online supplementary material, and websites) so that readers can easily obtain a complete description of the intervention.

Although reporting standards are necessary, they are unlikely to be sufficient. Development and enhancement of tools to assist 
with describing interventions would be useful. One such tool is PatPlot, ${ }^{19}$ which provides a graphic depiction of the elements and sequencing of complex interventions. However, given the documented substantial deficiencies in reporting of interventions and the demonstration that it is partly remediable, authors and editors should take action now to reduce this waste in research.

We thank lain Chalmers (editor, James Lind Library, Oxford, UK) and Susan Michie (professor of health psychology, University College London, UK) for reviewing the manuscript and providing helpful comments. We also thank the authors of included reports who responded to our questions.

Contributors: TCH and PPG were primarily responsible for study conception and design and for data analysis and interpretation. All authors had full access to all of the data in the study and take responsibility for the integrity of the data and the accuracy of the data analysis. CE assisted with acquisition of the data and administrative support. TCH led the writing of the first draft of the manuscript, and all authors contributed to drafting and revising the manuscript. TCH and $P P G$ are the guarantors.

Funding: $\mathrm{TCH}$ is supported by a National Health and Medical Research Council of Australia (NHMRC)/Primary Health Care Research Evaluation and Development Career Development Fellowship (number: 1033038), with funding provided by the Australian Department of Health and Ageing. PPG is supported by a NHMRC Australia Fellowship (number: 527500). The funders had no role in design and conduct of the study; collection, management, analysis, and interpretation of the data; and preparation, review, or approval of the manuscript.

Competing interests: All authors have completed the ICMJE uniform disclosure form at www.icmje.org/coi_disclosure.pdf (available on request from the corresponding author) and declare: $\mathrm{TCH}$ is supported by a NHMRC/Primary Health Care Research Evaluation and Development Career Development Fellowship, and PPG is supported by a NHMRC Australia Fellowship; no financial relationships with any organisations that might have an interest in the submitted work in the previous three years; no other relationships or activities that could appear to have influenced the submitted work.

Ethical approval: Not required.

Data sharing: Data on the included trials and their ratings are available on request from the corresponding author at

Tammy_Hoffmann@bond.edu.au.
1. Aronson JK. Patent medicines and secret remedies. BMJ 2009;339:b5415.

Duff JM, Leather H, Walden EO, LaPlant KD, George TJ. Adequacy of published oncology randomized controlled trials to provide therapeutic details needed for clinical application $J$ Natl Cancer Inst 2010;102:702-5.

3 Glasziou P, Meats E, Heneghan C, Shepperd S. What is missing from descriptions of treatment in trials and reviews? BMJ 2008;336:1472-4.

4 Boutron I, Moher D, Altman DG, Schulz KF, Ravaud P. Extending the CONSORT statement to randomised trials of nonpharmacologic treatment: explanation and elaboration. Ann Intern Med 2008;148:295-310.

5 Davidson KW, Goldstein M, Kaplan RM, Kaufmann PG, Knatterud GL, Orleans CT, et al. Evidence-based behavioral medicine: what is it and how do we achieve it? Ann Behav Med 2003;26:161-71.

6 Schroter S, Glasziou P, Heneghan C. Quality of descriptions of treatments: a review of published randomised controlled trials. BMJ Open 2012;2:1-7.

7 Chalmers I, Rounding C, Lock K. Descriptive survey of non-commercial randomised controlled trials in the United Kingdom, 1980-2002. BMJ 2003;327:1017.

8 Hupperets MD, Verhagen EA, van Mechelen W. Effect of unsupervised home based proprioceptive training on recurrences of ankle sprain: randomised controlled trial. $B M J$ 2009;339:b2684.

9 Pino C, Boutron I, Ravaud P. Inadequate description of educational interventions in ongoing randomised controlled trials. Trials 2012;13:63.

10 Robb SL, Carpenter JS. A review of music-based intervention reporting in paediatrics. J Health Psychol 2009;14:490-501.

11 Jacquier I, Boutron I, Moher D, Roy C, Ravaud P. The reporting of randomised clinica trials using a surgical intervention is in need of immediate improvement: a systematic review. Ann Surg 2006;244:677-83.

12 Chalmers I, Glasziou P. Avoidable waste in the production and reporting of research evidence. Lancet 2009;374:86-9.

13 Glasziou P, Chalmers I, Altman DG, Bastian H, Boutron I, Brice A, et al. Taking healthcare interventions from trial to practice. BMJ 2010;341:c3852.

14 Hopewell S, Altman DG, Moher D, Schulz KF. Endorsement of the CONSORT statement by high impact factor medical journals: a survey of journal editors and journal "instructions to authors". Trials 2008:9:20.

15 Glasziou PP. Promoting evidence-based non-drug interventions: time for a non-pharmacopoeia? Med J Aust 2009;191:52-3.

16 Glasziou P. Making non-drug interventions easier to find and use. Aust Fam Physician 2013;42:35.

17 Péron J, Pond GR, Gan HK, Chen EX, Almufti R, Maillet D, et al. Quality of reporting of modern randomised controlled trials in medical oncology: a systematic review. J Natl Cancer Inst 2012;104:982-9.

18 Chan A, Tetzlaff JM, Gøtzsche PC, Altman DG, Mann H, Berlin JA, et al. SPIRIT 2013 explanation and elaboration: guidance for protocols of clinical trials. BMJ 2013;346:e7586

19 Perera R, Heneghan C, Yudkin P. Graphical method for depicting randomised trials of complex interventions. BMJ 2007;334:127-9.

Accepted: 29 May 2013

\section{Cite this as: BMJ 2013;347:f3755}

This is an Open Access article distributed in accordance with the Creative Commons Attribution Non Commercial (CC BY-NC 3.0) license, which permits others to distribute, remix, adapt, build upon this work non-commercially, and license their derivative works on different terms, provided the original work is properly cited and the use is non-commercial. See: http://creativecommons.org/licenses/by-nc/3.0/. 


\section{What is already known on this topic}

Incomplete descriptions of interventions render the intervention uninterpretable and unusable by clinicians, patients, and researchers The completeness of descriptions of interventions may be worse for non-pharmacological interventions than for pharmacological interventions

\section{What this study adds}

Missing information is a common problem (occurring in more than $50 \%$ ) in reports of non-pharmacological interventions Details of intervention materials and procedures were the most commonly missing elements

The problem can be partially remediated by contacting authors of trial reports for missing details

\section{Tables}

Table 1 | Items in checklist used to assess reports of randomised trials of non-pharmacological interventions

\begin{tabular}{ll}
\begin{tabular}{ll} 
Checklist item & Elaboration of item \\
Setting & Is it clear where the intervention was delivered? \\
\hline Recipient & Is it clear who received the intervention, and do you know all that you need to know about the participants? \\
\hline Provider & Is it clear who delivered the intervention? \\
\hline Procedure & Is the procedure (including the sequencing of the technique) of the intervention sufficiently clear to allow replication? \\
\hline Materials & Are the physical or informational materials used adequately described (and available)? \\
\hline Intensity & Is the dose/length of individual sessions of the intervention clear? \\
\hline Schedule & Is the schedule (interval, frequency, duration, or timing) of the intervention clear? \\
\hline Missing (overall) & Is the description of the intervention complete?
\end{tabular} \\
\hline
\end{tabular}


Table 2| Publication source of trials $(n=133)$ and categories of interventions $(n=137)$ evaluated

\begin{tabular}{ll} 
Source/category & No (\%) \\
Journal & $39(29)$ \\
\hline BMJ & $29(22)$ \\
\hline JAMA & $23(17)$ \\
\hline New England Journal of Medicine & $22(17)$ \\
\hline Lancet & $15(11)$ \\
\hline Annals of Internal Medicine & $5(4)$ \\
\hline PLOS Medicine & \\
\hline Categories of interventions evaluated & $23(17)$ \\
\hline Education and training & $23(17)$ \\
\hline Device & $19(14)$ \\
\hline Surgery or perioperative intervention & $17(12)$ \\
\hline Complex intervention & $16(12)$ \\
\hline Diet & $15(11)$ \\
\hline Exercise or physical therapy & $9(7)$ \\
\hline Service delivery & $8(6)$ \\
\hline Other (such as sand as playground surface) & $6(4)$ \\
\hline Psychosocial intervention & $1(0.7)$ \\
\hline Complementary and alternative therapy &
\end{tabular}




\begin{tabular}{|c|c|c|}
\hline $\begin{array}{l}\text { Checklist } \\
\text { item }\end{array}$ & Verbatim examples of poor reporting ${ }^{*}$ & $\begin{array}{l}\text { Reason for initial rating as "Not reported or not clearly } \\
\text { described" }\end{array}$ \\
\hline Setting & $\begin{array}{l}\text { "We conducted a randomised, sham-controlled study involving } 24 \text { patients with stroke } \\
\text { ( } 11 \text { men and } 13 \text { women). The median age was } 62 \text { years (range, } 53 \text { to } 71 \text { ), and the } \\
\text { median time since stroke was } 14 \text { months (range, } 7 \text { to } 21 \text { )." }\end{array}$ & $\begin{array}{l}\text { No details given about setting of intervention-for example, } \\
\text { outpatient setting, community setting, or in participants' homes } \\
\text { (author clarified in an email) }\end{array}$ \\
\hline Provider & $\begin{array}{l}\text { "The exercise training consisted of } 36 \text { sessions of supervised aerobic exercise training } \\
\text { (ie, walking, treadmill, or stationary cycling) at } 60 \% \text { to } 70 \% \text { of heart rate reserve } 3 \\
\text { times per week followed by prescribed home-based training at the same intensity } 5 \\
\text { times per week." }\end{array}$ & $\begin{array}{l}\text { No details provided about who supervised training and their } \\
\text { role in supervising training }\end{array}$ \\
\hline \multirow[t]{3}{*}{ Procedure } & $\begin{array}{l}\text { "Patients in the intervention group followed a standardised exercise protocol tailored } \\
\text { to individual achievement and were supervised by a physical therapist. The } \\
\text { programme consisted of a general warm up on a bicycle ergometer followed by static } \\
\text { and dynamic muscular exercises for the quadriceps, adductor, and gluteal muscles. } \\
\text { The programme also included balance exercises and flexibility exercises for major } \\
\text { thigh muscles." }\end{array}$ & $\begin{array}{l}\text { Details of the procedure, including "standardised exercise } \\
\text { protocol" are not clear (author provided further details in an } \\
\text { email) }\end{array}$ \\
\hline & $\begin{array}{l}\text { "Behavioral counseling was integrated into the group and individual sessions to } \\
\text { promote adherence to the assigned diets." }\end{array}$ & $\begin{array}{l}\text { Details of the behavioural counselling not clear (author } \\
\text { provided further details in an email) }\end{array}$ \\
\hline & $\begin{array}{l}\text { "Based on previous research related to maternal dissatisfaction with peer support, } \\
\text { the peer volunteers were requested to make a minimum of four contacts and then } \\
\text { to interact as deemed necessary." }\end{array}$ & Procedure of intervention not clear \\
\hline \multirow[t]{3}{*}{ Materials } & $\begin{array}{l}\text { "Patients randomised to the intervention joined a manual based, self directed, physical } \\
\text { rehabilitation programme developed by physiotherapists and introduced by a study } \\
\text { nurse." }\end{array}$ & $\begin{array}{l}\text { Procedure of intervention not clear, and details about } \\
\text { accessing manual not provided in report (author provided } \\
\text { manual and details of procedure in an email) }\end{array}$ \\
\hline & "A 90-minute, semiscripted group session that was led by the genetic counsellor. . & $\begin{array}{l}\text { Neither script nor details about how to access it was provided } \\
\text { in report }\end{array}$ \\
\hline & $\begin{array}{l}\text { "We offered two half day training seminars for } 20 \text { health professionals in each locality: } \\
\text { one on group facilitation skills led by an external consultant and one on trial conduct, } \\
\text { protocol, and data collection. We provided a written training pack and a password } \\
\text { protected website with access to all training materials." }\end{array}$ & $\begin{array}{l}\text { No details about accessing training materials were provided } \\
\text { in paper (authors provided materials after request via email) }\end{array}$ \\
\hline \multirow[t]{2}{*}{ Intensity } & "The intervention was delivered by 1 nurse during bimonthly telephone calls." & $\begin{array}{l}\text { Duration of telephone calls (planned or actual) not reported } \\
\text { (author clarified in an email) }\end{array}$ \\
\hline & $\begin{array}{l}\text { We offered participants assigned to moderate-intensity disease management up to } \\
2 \text { telephone-based counselling sessions every } 6 \text { months (Ellerbeck) }\end{array}$ & Length of counselling session not known \\
\hline \multirow[t]{2}{*}{ Schedule } & $\begin{array}{l}\text { "Each encounter included a core group of modules ... plus additional modules } \\
\text { activated at specific intervals." }\end{array}$ & $\begin{array}{l}\text { "Specific intervals" are not provided (author clarified details } \\
\text { in an email) }\end{array}$ \\
\hline & $\begin{array}{l}\text { "Nutritionists and dietitians gave dietary advice to participants in both groups in } \\
\text { monthly sessions in the first year and bimonthly sessions thereafter." }\end{array}$ & $\begin{array}{l}\text { Not clear how long bimonthly sessions continued for (follow-up } \\
\text { in trial was } 4 \text { years) }\end{array}$ \\
\hline
\end{tabular}




\section{Figures}

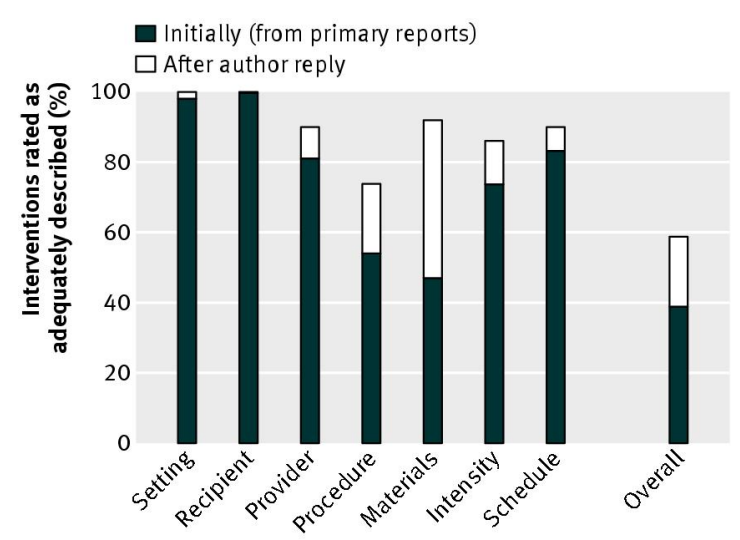

Fig 1 Percentage of interventions rated as adequately described, in primary report and after author reply, for each checklist item

Excerpt of intervention descriptionsfrom original paper

tested in a pilot study. A detailed description of the different basic exercises of the proprioceptive training programme is described elsewhere, ${ }^{x}$ and the training programme is in the appendix on bmj.com. The pro gramme prescribed three training sessions a week, with a maximum duration of 30 minutes a session. Athletes were encouraged to perform the exercises as part of their normal warm up. Exercises gradually increased in difficulty and training load during the eight week programme

Athletes in the intervention group received a bal ance board (Avanco AB, Sweden), exercise sheets, and an instructional DVD showing all exercises of the programme. All information was also provided on a website, accessible only for those in the intervention group.

\section{Examples of materials a vailable}

\section{Web Extra}

citra nateral suppled by the autor

Files in this Dosta Supplement:

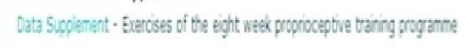

Versterk je enkel

van Consument en Veiligheid

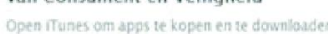

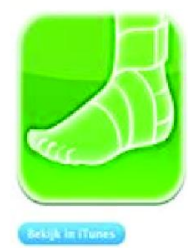

Omschrijving

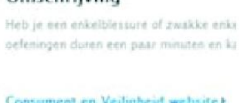

Wat is nieuw in versie 1.2

Oocomatsme voun ios 5

\footnotetext{
Questions sent to author and their responses in quotation marks

1. a) Is the written training pack and DVD publicly available and if so, where from?

"Yes, online (www.sportzorg.nl) or in an android and iPhone app (Enkel)."

b) If not are you able to send a copy to us please?

"If required, yes".

2. Was there an initial face-to-face meeting where the exercise were explained?

"No. The intervention was to be unsupervised and individually".

3. Who gave the material to patients and were any verbal instructions given as well?

"The primary researcher. No verbal instructions were given".

4. Was there any support available during the intervention period?

"Yes, if needed researchers could be reached by phone".

5. Can you give details how the grading of exercise over the 8 weeks was done? Was it

tailored for each patient or standardized in the exercise program?

"Standardized through an exercise schedule". (NB: Schedule is clear in the iPhone app)
}

Fig 2 Illustration of process of obtaining complete description of an intervention (from: Effect of unsupervised home based proprioceptive training on recurrences of ankle sprain: randomised controlled trial ${ }^{8}$ ) 


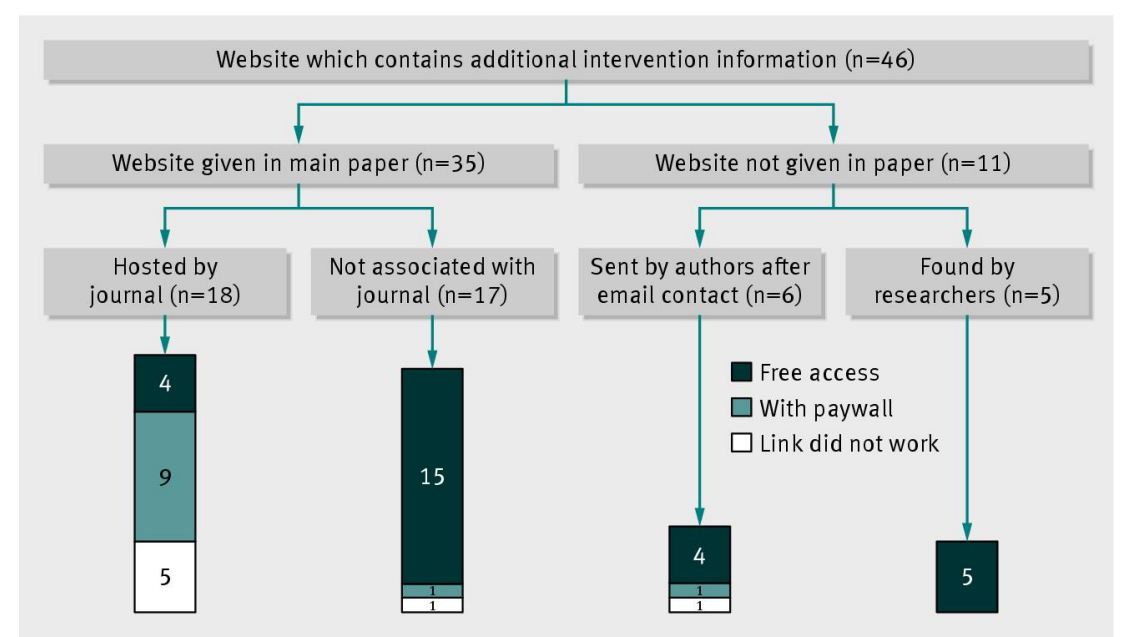

Fig 3 Access to and source of websites that contained additional intervention information 